\title{
昡pubvet
}

https://doi.org/10.22256/pubvet.v12n4a73.1-5

\section{Avaliação de corante fluorescente benzazólico para detecção em parasitos diagnosticados por flutuação simples}

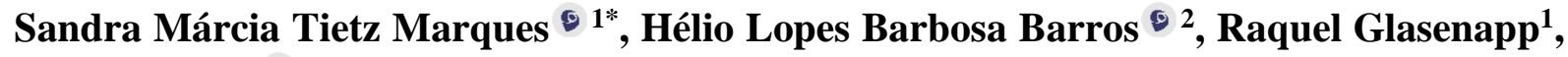 \\ Valter Stefani ${ }^{\bullet 2}$ \\ ${ }^{1}$ Laboratório de Helmintoses, Departamento de Patologia Clínica Veterinária, Universidade Federal do Rio Grande do Sul, Porto Alegre, RS, \\ Brasil.E-mail: smtmuni@hotmail.com; raquelglasenapp@hotmail.com \\ ${ }^{2}$ Laboratório de Novos Materiais Orgânicos e Ciência Química Forense, Instituto de Química, Universidade Federal do Rio Grande do Sul, \\ Porto Alegre, RS Brasil.E-mail: h.barros@campus.fct.unl.pt; vstefani@gmail.com \\ *Autor para correspondência
}

RESUMO. Este estudo mostra o corante fluorescente 4-hidroxi-3- (6metilbenzo[d]oxazol-2-il) benzeno sulfônico (HB-9) marcando, por microscopia de fluorescência, ovos de helmintos e oocistos de protozoários diagnosticados em animais domésticos. Foram selecionados ovos de Acuaria spiralis, Strongyloides papillosus, Capillaria spp., Bertiella spp., Strongyloidea e Eimeria spp., identificados previamente pelo método de flutuação. As amostras foram observadas em microscópio BEL Photonics INV-100 FL Plus com sistema de Epi-Fluorescência. Ovos de A. spiralis, Capillaria spp., Bertiella spp. e Eimeria spp. apresentaram fluorescência. O corante mostrou ser capaz de marcar as estruturas parasitárias de modo seletivo, facilitando a visualização do material de interesse.

Palavras chave: benzazola, biomarcadores, corante fluorescente, parasitos

\section{Evaluation of benzazolic fluorescent dye for the detection of parasites diagnosed by simple flotation}

ABSTRACT. This study shows the use of 4-hydroxy-3- (6-methylbenzo [d] oxazol-2-yl) benzenesulfonic acid (HB-9), a fluorescent dye, for the detection and visualization of helminth eggs and protozoan oocysts diagnosed in domestic animals, by epifluorescence microscopy. Eggs of Acuaria spiralis, Strongyloides papillosus, Capillaria spp., Bertiella spp., Strongyloidea and Eimeria spp., were previously identified through the flotation method and used for analysis. The fluorescence of the eggs was visualized in a BEL R Photonics com sistema de Epi-FluorescênciaINV-100 FL Plus..Only eggs of Acuaria spiralis, Capillaria spp., and Bertiella spp. presented fluorescence when visualized by epifluorescence microscopy. The results indicate that the fluorescente dye used in this study permits a easy, clear and selective detection and visualization ofparasitic structures.

Keywords: Benzazole, biomarkers, fluorescent dye, parasites

\section{Evaluación de colorante fluorescente benzazolico para detección en parásitos diagnosticados por flotación simple}

RESUMEN. Este estudio muestra el colorante fluorescente 4-hidroxi-3- (6-metilbenzo [d] oxazol-2-il) benceno sulfónico (HB-9) marcando, por microscopía de fluorescencia, huevos de helmintos y oocistos de protozoarios diagnosticados en animales domésticos. Fueron seleccionados huevos de Acuaria spiralis, Strongyloides papillosus, Capillaria 
spp., Bertiella spp., Strongyloidea y Eimeria spp., identificados previamente por el método de fluctuación. Las muestras fueran observadas en microscopio BEL Photonics INV-100 FL Plus con sistema de Epi-Fluorescencia. Huevos de Acuaria spiralis, Capillaria spp., Bertiella spp. y Eimeria spp. presentaron fluorescencia. El colorante mostró ser capaz de marcar las estructuras parasitarias de modo selectivo, facilitando la visualización del material de interés.

Palabras clave: Benzazola, biomarcadores, colorante fluorescente, parásitos

\section{Introdução}

As doenças parasitárias são seculares, cosmopolitas, de prevalência variada, algumas reemergentes acometendo animais e seres humanos. A Organização Mundial de Saúde relata que as parasitoses humanas são responsáveis por dois a três milhões de óbitos por ano no mundo, e cerca de 84 milhões de indivíduos infectados por Ascaris lumbricoides e mais de 100 milhões por Trichuris trichiura e ancilostomídeos (Hotez et al., 2008). Em termos globais, aproximadamente 300 milhões de pessoas sofrem algum grau de severidade, entretanto o maior impacto das helmintíases transmitidas pelo solo decorre do mau desenvolvimento fisico e mental em crianças (WHO, 2010). Em animais domésticos e silvestres tem relevância em saúde pública pelo caráter zoonótico. Os parasitos ocorrem praticamente em todos os níveis tróficos e sua transmissão pode depender da presença de uma variedade de hospedeiros intermediários, paratênicos e definitivos dentro do ecossistema. Essa complexidade de fatores exerce importantes efeitos sobre as populações de seus hospedeiros, alterando o comportamento, sucesso reprodutivo e mortalidade. Os efeitos também são econômicos, e muitas espécies animais silvestres são considerados importantes indicadores ambientais (Godoy \& Cubas, 2011) e podem representar uma ameaça para os programas de manejo e recuperação de populações, de especial importância para espécies ameaçadas. Por isso, a identificação da fauna parasitária é exigida em protocolos de reintrodução e na rotina clínica de animais silvestres (Godoy \& Cubas, 2011, Bandelj et al., 2015, Santos et al., 2015).

Fluoróforos são compostos de ampla aplicação tecnológica, uma vez que possuem grande sensibilidade de detecção e são facilmente observáveis. $\mathrm{Na}$ área de sondas biológicas, possui potencialidades apreciáveis, pois facilita e acelera as leituras microscópicas. Compostos heterocíclicos benzazólicos apresentam intensa emissão de fluorescência, além de alta estabilidade térmica e fotoquímica, antes e depois de sua utilização. Estes compostos têm mostrado diferentes atividades farmacológicas, tais como antiviral (Song et al., 2005), antibiótico (Evans et al., 1979), antifungo (Yamato, 1992), anticancer (Kumar et al., 2002), antimicrobial (Yildiz-Oren et al., 2004) e anti-Parkinson (Benazzouz et al., 1995). Nós relatamos aqui o uso de um corante fluorescente incorporando grupos hidrofílicos que aumentam a solubilidade em água e reatividade com biomoléculas, já relatados previamente no diagnóstico de oxuriase equina pelo teste de Graham (Barros et al., 2016, Barros \& Stefani, 2016a). Dentre suas diversas aplicações possíveis, está a coloração fluorimétrica de amostras parasitológicas para visualização rápida e precisa de cistos, ovos e larvas de helmintos e protozoários de interesse para diagnóstico e/ou de pesquisa. Diferente das técnicas imunofluorimétricas, os corantes sintetizados aderem diretamente aos parasitos, o que possibilitaria seu uso em rotina clínica. O diagnóstico parasitológico através de técnicas apropriadas, com rapidez, sensibilidade, especificidade e custo baixo são fundamentais para fazer frente aos desafios de controle e poder monitor as taxas de infecção com rapidez e custo baixo. Portanto o objetivo deste estudo é avaliar a fixação do corante fluorescente 4-hidroxi-3-(6metilbenzo[d]oxazol-2-1)benzeno sulfônico (HB9) na visualização de estruturas parasitárias em microscopia de fluorescência, facilitando o diagnóstico parasitológico.

\section{Material e Métodos}

Procedimentos gerais para síntese de benzazol fluorescente solúvel em água

Todos os reagentes e solventes utilizados na síntese foram adquiridos na Sigma-Aldrich, Acros ou Merck e foram usados sem qualquer purificação. Os espectros de infravermelho foram registrados em um Mattson Galaxy Series FT-IR 3000 em KBr. A síntese do composto foi obtida conforme Barros \& Stefani (2016b), patente número BR102014030942, descrito como 4hidroxi-3-(6-metilbenzo[d]oxazol-2-il) benzeno sulfônico (HB-9). 
Exames coprológicos pelo método de flutuação e análises microscópicas

Amostras de fezes de rouxinol (Luscinia megarhynchos), ovinos, galinha e bugio-ruivo (Alouatta guariba clamitans) recebidas para exame parasitológico de fezes (EPF) no Laboratório de Helmintoses da Faculdade de Veterinária da Universidade Federal do Rio Grande do Sul (UFRGS) foram processadas no prazo máximo de 24 horas do recebimento. O método diagnóstico se baseia no princípio da flutuação com solução saturada de cloreto de sódio. Os ovos, cistos e oocistos foram identificados de acordo com suas características morfológicas (Bowman, 2010, Hendrix \& Robinson, 2016). O material foi examinado em microscópio óptico (MO) marca Olympus CX 40, com objetivas de 10X, 20X e 40X. Após a visualização em $\mathrm{MO}$, as amostras positivas foram selecionadas e observadas em microscópio BEL Photonics INV-100 FL Plus com sistema de EpiFluorescência (MF) com filtros Verde, Azul,
Violeta e Ultravioleta e equipado com câmera CCD Blacklight. No momento da leitura da lâmina, acrescentou-se $100 \mu \mathrm{l}$ da solução HB-9. Primeiramente, o material é examinado usando objetiva de 10X e de 40X com luz reduzida. As amostras positivas em $\mathrm{MO}$ foram observadas em seguida por MF.

\section{Resultados e Discussão}

Foram diagnosticados ovos de Acuaria spiralis em rouxinol (Figura 1), Capillaria spp. em galinha (Figura 2), oocistos de Eimeria spp. e ovos de Bertiella spp. em bugio-ruivo (Figura 3), Strongyloides papillosus e ovos tipo Strongyloidea em fezes de ovinos (Figura 4).

Ovos de Acuaria spiralis, Capillaria spp., Bertiella spp. e oocistos de Eimeria spp. apresentaram fluorescência e melhor visualização nos filtros Ultravioleta e Azul. Foi possível observar a presença da larva em Acuaria spiralis demonstrando que o corante HB-9 foi capaz de atravessar a parede de $A$. spiralis.
A

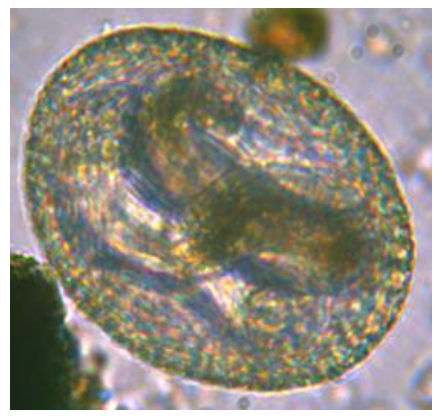

$\mathrm{B}$

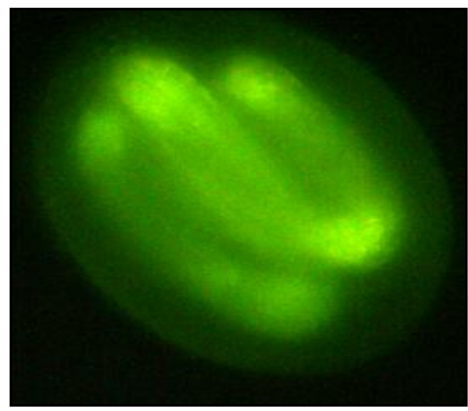

$\mathrm{C}$

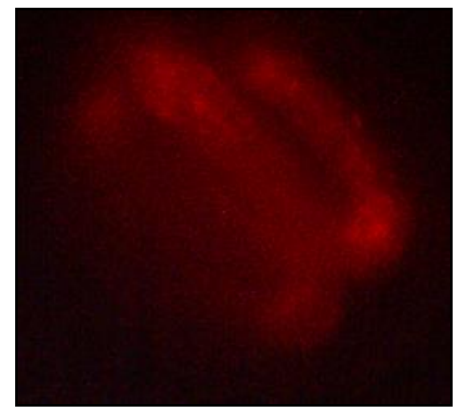

Figura 1. Imagens de microscopia óptica (A) e de epifluorescência (B - filtro azul e C-filtro verde) de ovos de Acuaria spiralis, após marcação com o corante HB- 9 na concentração de $1 \mathrm{mM}$ e aumento de $20 \mathrm{X}$.

A

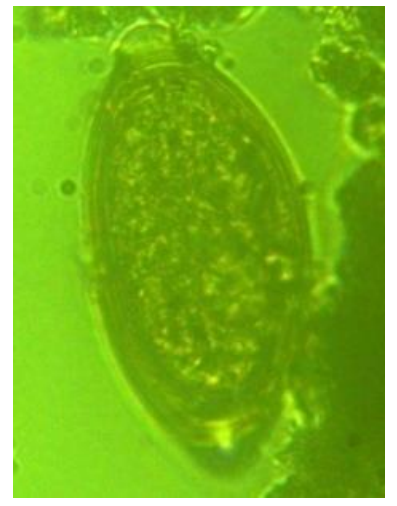

B

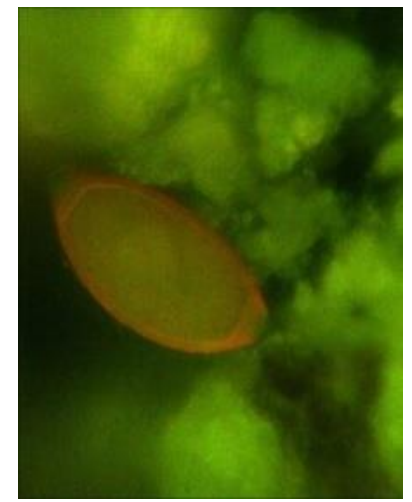

C

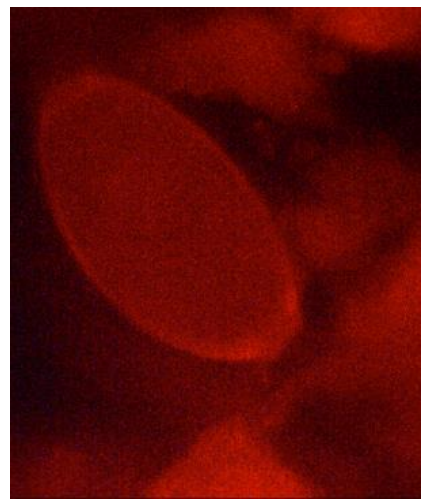

Figura 2. Imagens de microscopia óptica (A) e de epifluorescência (B - filtro ultravioleta e C-filtro verde) de ovos de Capillaria spp, após marcação com o corante HB-9 na concentração de 1mM. Aumento de 40X (A/C) e 20X (B). 
A

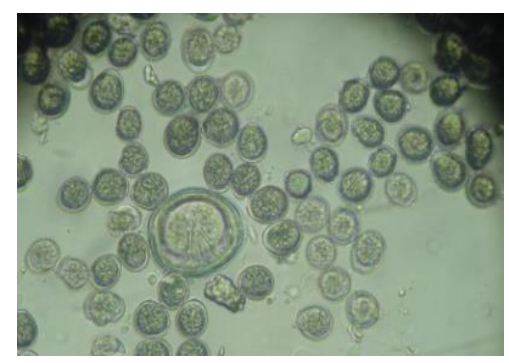

B

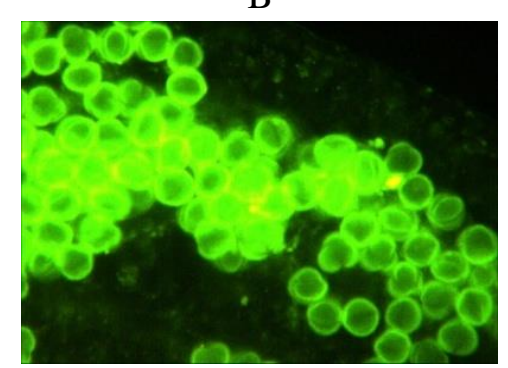

$\mathrm{C}$

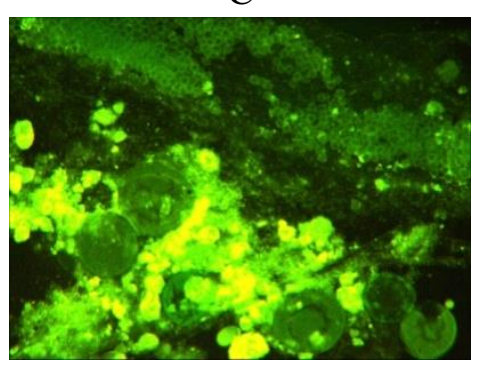

Figura 3. Imagens de microscopia óptica de oocisto de Eimeria spp. (A) e de epifluorescência de oocistos de Eimeria spp. (B) e Bertiella spp. (C), após marcação com corante HB-9 na concentração de 1 mM. Filtro UV e aumento de 10X.

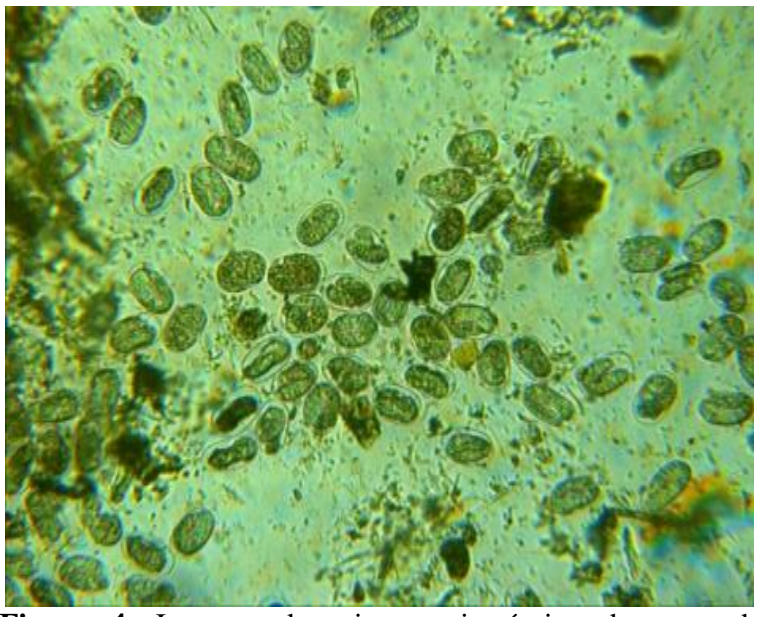

Figura 4. Imagem de microscopia óptica de ovos de Strongyloides papillosus e Strongyloidea pela técnica de Willis (aumento 10X).

$\mathrm{O}$ corante mostrou ser capaz de marcar às estruturas parasitárias de modo seletivo, facilitando a visualização do material de interesse Ovos tipo Strongyloidea e Strongyloides papillosus presentes na amostra fecal de ovino não foram visíveis sob microscopia de fluorescência. Os ovos de Strongyloides papillosus apresentamse embrionados por larva infectante - L1 - (assim como ovos de A. spiralis). Entretanto o corante HB-9 não marcou a parede dos ovos ou atravessou-a, porém marcou L1 bem como a parede de A. spiralis. Não podemos explicar, ainda, a falta de marcação, embora as amostras fecais de ovinos ou de rouxinol eram frescas, foram recebidas e processadas no mesmo dia. Talvez outros constituintes presentes nas fezes, ou o aumento na concentração ou no $\mathrm{pH}$ do corante possam alterar este resultado. De outro modo, mostrou seletividade, não corando debris, esporos de fungos, por exemplo, que normalmente estão presentes na flutuação obtida com material fecal. No entanto, o fato de outras estruturas tornaremse fluorescentes não representa uma desvantagem do método, pois a morfologia dos ovos e oocistos é facilmente distinguível.
Não houve diferença na qualidade das estruturas parasitárias visualizadas quando a solução era gotejada diretamente na lâmina, no momento da análise, imediatamente após a análise por MO, porque o corante não reage de modo especial com a solução saturada de $\mathrm{NaCl}$ (densidade $=1.20)$. Um tempo de um minuto é suficiente para a visualização. Algumas espécies de helmintos não coraram, possivelmente devido a diferenças em suas estruturas. Os fluoróforos usados apresentam vantagens sobre a imunofluorescência pois não necessitam de anticorpos para aderência nos parasitos, sua realização é relativamente simples e barata. No caso dos coccídeos detectados e avaliados neste estudo, não foram identificados ao nível das espécies, embora as amostras positivas foram posteriormente submetidas à esporulação. $\mathrm{O}$ tamanho, forma, cor, textura e tipo de conteúdo interno são importantes características utilizadas na identificação de oocistos de coccídios. Em estudos posteriores serão avaliados oocistos esporulados na tentativa de caracterizar os esporocistos e a presença ou não do corpo Stieda, um tampão proteináceo encontrado em uma extremidade do esporocisto.

\section{Conclusão}

O novo corante benzazólico HB-9 apresentou um bom resultado na marcação de helmintos e oocistos de protozoários, diagnosticados em animais domésticos. Todos os novos benzazóis sintetizados no presente artigo são bastante solúveis em água e apresentam fluorescência quando expostos à luz UV-visível $365 \mathrm{~nm}$, alta estabilidade química e fotoquímica, baixo custo e robustez, pois não depende da temperatura, $\mathrm{pH}$, abrigo da luz. Diferença de cor, intensidade e homogeneidade de fluorescência foram observadas quando usado diferentes tipos de filtros. 
Estes resultados mostram o potencial de utilização do HB-9 como sonda biológica na marcação de ovos e oocistos de parasitos. Estudo de tempo de impregnação, concentração e quantidade de corante são alguns pontos que devem ainda ser avaliados e determinar ao nível de gênero parasitário com precisão, rapidez e baixo custo, para ser utilizado na rotina. Estes resultados são significantes, indicando perspectivas de estudo para o desenvolvimento de um novo método para o diagnóstico laboratorial.

\section{Referências Bibliográficas}

Bandelj, P., Blagus, R., Trilar, T., Vengust, M. \& Rataj, A. V. 2015. Influence of phylogeny, migration and type of diet on the presence of intestinal parasites in the faeces of European passerine birds (Passeriformes). Wildlife biology, 21, 227-233.

Barros, H. L., Marques, S. M. \& Stefani, V. 2016. The use of epifluorescence microscopy and fluorescent dyes for visualization of Oxyuris equi eggs. Veterinary Parasitology, 226, 162166.

Barros, H. L. \& Stefani, V. 2016a. A new methodology for the visualization of latent fingermarks on the sticky side of adhesive tapes using novel fluorescent dyes. Forensic Science International, 263, 83-91.

Barros, H. L. B. \& Stefani, V. 2016b. Método de obtenção de derivados benzazólicos fluorescentes e sua aplicação em ciência forense como reveladores de impressões digitais latentes.

Benazzouz, A., Boraud, T., Dubédat, P., Boireau, A., Stutzmann, J.-M. \& Gross, C. 1995. Riluzole prevents MPTP-induced parkinsonism in the rhesus monkey: a pilot study. European Journal of Pharmacology, 284, 299-307.

Bowman, D. D. 2010. Parasitologia veterinária. Elsevier.

Evans, D. A., Sacks, C. E., Kleschick, W. A. \& Taber, T. R. 1979. Polyether antibiotics synthesis. Total synthesis and absolute configuration of the ionophore A-23187. Journal of the American Chemical Society, 101, 6789-6791.

Godoy, S. N. \& Cubas, Z. S. 2011. Doenças virais e parasitárias em Psittaciformes: uma revisão. Clínical Veterinária, 90, 32-44.
Hendrix, C. M. \& Robinson, E. D. 2016. Diagnostic Parasitology for Veterinary Technicians. Elsevier Health Sciences, Saint Louis, USA.

Hotez, P. J., Bottazzi, M. E., Franco-Paredes, C., Ault, S. K. \& Periago, M. R. 2008. The neglected tropical diseases of Latin America and the Caribbean: a review of disease burden and distribution and a roadmap for control and elimination. PLoS Neglected Tropical Diseases, 2, e300.

Kumar, D., Jacob, M. R., Reynolds, M. B. \& Kerwin, S. M. 2002. Synthesis and evaluation of anticancer benzoxazoles and benzimidazoles related to UK-1. Bioorganic \& Medicinal Chemistry, 10, 3997-4004.

Santos, P. M. S., Silva, S. G. N., Fonseca, C. F. \& Oliveira, J. B. 2015. Parasitos de aves e mamíferos silvestres em cativeiro no estado de Pernambuco. Pesquisa Veterinária Brasileira, 35, 788-194.

Song, X., Vig, B. S., Lorenzi, P. L., Drach, J. C., Townsend, L. B. \& Amidon, G. L. 2005. Amino acid ester prodrugs of the antiviral agent 2-bromo-5, 6-dichloro-1-( $\beta$-Dribofuranosyl) benzimidazole as potential substrates of hPEPT1 transporter. Journal of Medicinal Chemistry, 48, 1274-1277.

WHO. 2010. World Health Organization. Strategies to monitor and evaluate population sodium consumption and sources of sodium in the diet. In: Canada, G. o. (ed.) WHO technical report series. Canada.

Yamato, M. 1992. Study on the development of biological-active compounds after the model of natural products. Yakugaku zasshi: Journal of the Pharmaceutical Society of Japan, 112, 8199.

Yildiz-Oren, I., Yalcin, I., Aki-Sener, E. \& Ucarturk, N. 2004. Synthesis and structureactivity relationships of new antimicrobial active multisubstituted benzazole derivatives. European Journal of Medicinal Chemistry, 39, 291-298.

Article History:

Received 30 December 2017

Accepted 16 February 2018

Available online 30 March 2018

License information: This is an open-access article distributed under the terms of the Creative Commons Attribution License 4.0, which permits unrestricted use, distribution, and reproduction in any medium, provided the original work is properly cited. 